Article

\title{
Study of Flow between Non-Isothermal Disks in Hydro-Viscous Drive
}

\author{
Jianzhong Cui* \\ School of Mechanical Engineering, Yancheng Institute of Technology, Yancheng, P. R. China \\ *Corresponding author: Jianzhong Cui (cuijianzhong21@163.com)
}

Manuscript received 11 October 2017; accepted 12 June 2018; published 31 July 2018

\begin{abstract}
The film flow between the disks is the critical factor in the design of hydro-viscous drive. In the previous work dealing with the flow in the gap, few authors considered the effect of non-isothermal interface on the flow. In order to investigate the dynamic behavior of the oil film between non-isothermal friction pair in hydro-viscous drive, the flow field of the oil film between the disks is presented with consideration of four representative temperature models. Parameters related to the flow, such as temperature, pressure, velocity, shear stress and viscous torque are obtained by means of numerical simulation. The results show that non-isothermal interface results in temperature distribution profiles that diverge from the ideal parabolic curve. Both the discrepancies about film pressure and radial velocity at the outlet are vulnerable to temperature variation derived from a set of frictional pair. Tangential velocity profiles diverge from inlet to outlet due to the non-isothermal interface. Shear stress profiles show the opposite trend caused by the substantial variation about the oil viscosity. The research results lay a theoretical foundation for the reasonable designs of hydro-viscous drive.
\end{abstract}

\section{Keywords}

hydro-viscous drive, numerical analysis, oil film, non-isothermal interface, computational fluid dynamics(CFD)

\section{Introduction}

Hydro-viscous drive, HVD for short, is one of the most essential elements of controlled start transmission system and plays a crucial role in the soft-start and speed-regulating process. Historically, as HVD employs the oil film shear force between multidisc friction pairs to transmit torque from input shaft to output shaft, the most common HVD are used for direct connection of coaxial shafts in the industrial sector equipped with high power water pumps or fan speed governing system. Until recently, the continuously hydro-viscous drive is gradually being highly integrated into environment equipment or tunnel engineering equipment such as the wind turbine drive trains and tunnel boring machines with flexible control characteristics [1]. Future demands and tendencies in technology of producing friction pairs make many requirements referring their structure, functioning, strength or life. For this reason, the basic problem about the flow field of oil film between the disks should be sufficiently investigated.

The analysis of temperature for clutch discs has been performed in order to better understand the thermal failure behavior in the past. Several three-dimensional transient thermo-mechanical coupled models were developed, and some finite element simulations were performed to obtain temperature fields of the friction surfaces in clutches (Cui, J. Z. et al. [2]; Yevtushenko A. et al. [3]; Liu, J. et al. [4]). It was found that high thermal stress occurs due to the considerable temperature variation during operation. More importantly, numerous researches have been conducted on the flow field of oil film between the disks. Davis, C. L. et al. [5] extended the simplified isothermal model for wet clutch engagement developed by Berger, E. J. et al. [6] to include fluid thermal effects. The effect of temperature on the torque transfer and lubricant temperature variation was obtained. Yuan, Y. Q. et al. [7] simulated the flow within the disengaged wet clutch packs of an automatic transmission using the commercial computational fluid dynamics (CFD) code FLUENT. Aphale, C. R. et al. [8] found that the relative motion of the friction and separator disks resulted in a drag torque on both the disks that wasted energy and decreased fuel economy. An accurate mathematical model for the problem with verification using FLUENT and experiments was developed. Yuan, Y. Q. et al. [9] presented a mathematical model describing the hydrodynamics of the flow within a disengaged wet transmission clutch. The primary improvement of this model over the existing ones was the inclusion of the surface tension. Huang, J. H. et al [10, 11] investigated the flow field of the oil film between frictional pairs in hydro-viscous drive test rig. Temperature was found to have influence on the dynamic characteristics of the oil film. Furthermore, the simplified mathematical model based on steady and laminar flow considering the effect of variable viscosity was solved by means of computational fluid dynamics 
code FLUENT. Xie, F. W. et al. [12] derived the analytical solutions for pressure, radial velocity and transferred torque with consideration of temperature and the effect of pressure difference and inertia force on oil film. Manshoor, B. et al. [13] investigated the three dimensional CFD analysis regarding the performance of a thin film lubricated journal bearing. Three turbulent models were used to simulate characteristics of a plain journal bearing. Lin, Q. Y. et al. [14] applied a new transient analysis method combining computational fluid dynamics and fluid-structure interaction based on actual physical model. Furthermore, both thermal influence and cavitation were studied. Li, M. et al. [15] performed a three-dimensional thermohydrodynamic analysis of a wet clutch that covered the entire cycle of engagement from slip to lock to detachment. Xiong, C. B. et al. [16] investigated the thermal problem of plates' slid against symmetrically located contacting pads. Finite element method of moving heat input was used to calculate the temperature field which was validated by comparing with some existing analytical solutions. Zhang, Z. G. et al. [17] established the friction transmission model of wet clutch to analyze the friction transmission mechanism of its engagement. The onedimension transient model of heat transfer in radial direction was built on the basis of the heat transfer theory and the conservation law of energy. Cho, J. et al. [18] combined a new iterative scheme and commercial CFD software to simulate the squeeze film process in a wet clutch. It was found that the choice of boundary conditions had a strong influence on squeeze film simulation. Noh, D. et al. [19] developed an analysis model for predicting the lubricant flow rate. The reliability of the model was verified by comparing its predictions for various operation conditions with experimental measurements.

In general, the oil film between the disks provides lubrication and carries lots of heat away to avoid thermal failure of the disks. However, in the absence of sufficient cooling, the high face-temperature rise can lead to oxidation and decomposition of the oil film, which exercises a great influence on dynamic transmission during the soft-start. Previous research works are mainly concentrated on the simulation results of flow field between a pair of isothermal disks. It is assumed that the oil temperature near the wall surface is the same as that at the inlet. However, during the engagement of multidisc friction pairs employed in dynamic transmissions, the driving disk in a set of friction pair is also the driven disk in the adjacent set of friction pair. Furthermore, there is some difference about temperature distribution between any two sets of friction pairs. Therefore it is unrealistic to take for granted that temperature distribution along $r$-direction of oil film adjacent to all the disks can be assumed to be the same.

In the present work, we focus our attention on the dynamic behavior of the oil film between non-isothermal friction pair in hydro-viscous drive, as compared to isothermal friction pair. The flow field of the oil film between the disks is modelled and solved by using computational fluid dynamics (CFD) code, with consideration of four forms of representative temperature models about the disks. And the relationship between the oil temperature and its viscosity is taken into account. Extensive set of simulations and experiments are presented to examine influence of temperature and shear rate on the performance of HVD.

\section{Physical model}

The physical model of flow between the disks is shown

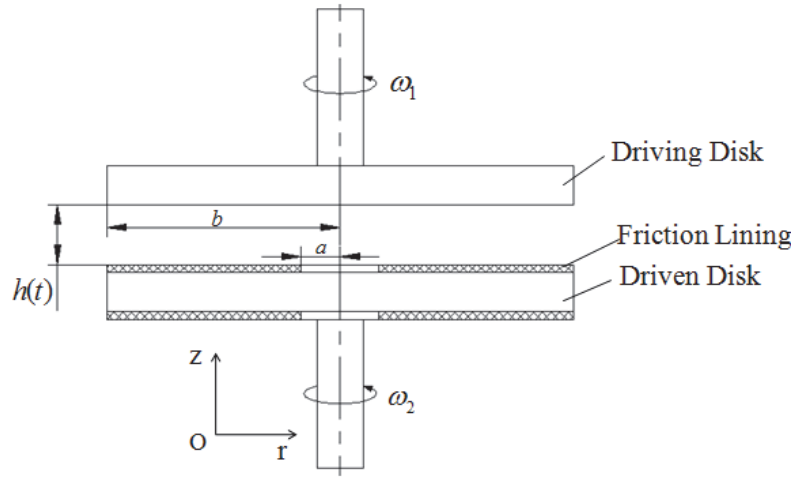

Fig. 1 Schematic of the HVD

schematically in Fig. 1. The driving disk rotates at a constant angular velocity $\omega_{1}$ and the driven disk rotates at an angular velocity $\omega_{2}$. The gap $h(t)$ is constant along the radial distance $r$, which is measured from the center of the annuli, is assumed to be completely filled with an incompressible viscous fluid. In the soft-start process, oil film is forced into the gap between the two disks when the disks are pushed together and the oil is squeezed out of the gap by integrated effect of pressure gradient and centrifugal force. The lubricant plays a role in transferring torque and cooling down the temperature. The transferred viscous torque across HVD can be calculated by Eq. (1), which is derived from Newton's law of viscosity [10].

$$
T_{h}=\frac{\pi \eta\left(\omega_{1}-\omega_{2}\right)}{2 h}\left(b^{4}-a^{4}\right)
$$

where $\eta$ is the dynamic viscosity of fluid.

\subsection{Governing equations}

The numerical solutions in this work correspond to simple rotating disk configurations. Under steady-state and axisymmetric flow conditions, the associated fluid mechanics problem about the soft-start process can be formulated in cylindrical coordinate. The governing equations are as follows.

Continuity equation

$$
\frac{\partial v_{r}}{\partial r}+\frac{1}{r} \frac{\partial v_{\theta}}{\partial \theta}+\frac{\partial v_{z}}{\partial z}+\frac{v_{r}}{r}=0
$$

Navier-Stokes equations

$$
\left\{\begin{array}{l}
\rho\left(\frac{\partial v_{r}}{\partial t}+v_{r} \frac{\partial v_{r}}{\partial r}+\frac{v_{\theta}}{r} \frac{\partial v_{r}}{\partial \theta}+v_{z} \frac{\partial v_{r}}{\partial z}-\frac{v_{\theta}^{2}}{r}\right)= \\
\rho F_{r}-\frac{\partial p}{\partial r}+\eta\left(\frac{\partial^{2} v_{r}}{\partial r^{2}}+\frac{1}{r} \frac{\partial v_{r}}{\partial r}+\frac{1}{r^{2}} \frac{\partial^{2} v_{r}}{\partial \theta^{2}}+\frac{\partial^{2} v_{r}}{\partial z^{2}}-\frac{2}{r^{2}} \frac{\partial v_{\theta}}{\partial \theta}-\frac{v_{r}}{r^{2}}\right) \\
\rho\left(\frac{\partial v_{\theta}}{\partial t}+v_{r} \frac{\partial v_{\theta}}{\partial r}+\frac{v_{\theta}}{r} \frac{\partial v_{\theta}}{\partial \theta}+v_{z} \frac{\partial v_{\theta}}{\partial z}+\frac{v_{r} v_{\theta}}{r}\right)= \\
\rho F_{\theta}-\frac{1}{r} \frac{\partial p}{\partial \theta}+\eta\left(\frac{\partial^{2} v_{\theta}}{\partial r^{2}}+\frac{1}{r} \frac{\partial v_{\theta}}{\partial r}+\frac{1}{r^{2}} \frac{\partial^{2} v_{\theta}}{\partial \theta^{2}}+\frac{\partial^{2} v_{\theta}}{\partial z^{2}}+\frac{2}{r^{2}} \frac{\partial v_{\theta}}{\partial \theta}+\frac{v_{\theta}}{r^{2}}\right) \\
\rho\left(\frac{\partial v_{z}}{\partial t}+v_{r} \frac{\partial v_{z}}{\partial r}+\frac{v_{\theta}}{r} \frac{\partial v_{z}}{\partial \theta}+v_{z} \frac{\partial v_{z}}{\partial z}\right)= \\
\rho F_{z}-\frac{\partial p}{\partial z}+\eta\left(\frac{\partial^{2} v_{z}}{\partial r^{2}}+\frac{1}{r} \frac{\partial v_{z}}{\partial r}+\frac{1}{r^{2}} \frac{\partial^{2} v_{z}}{\partial \theta^{2}}+\frac{\partial^{2} v_{z}}{\partial z^{2}}\right)
\end{array}\right.
$$

where $\rho$ is the oil density, $p$ is the oil pressure.

The conservation of energy equation for the oil film can be written as follows 
Table 1 Viscosity of $8^{\sharp}$ hydrodynamic transmission oil

\begin{tabular}{lcccccccc}
\hline Temperature $\left({ }^{\circ} \mathrm{C}\right)$ & 25 & 30 & 40 & 50 & 60 & 70 & 80 & 90 \\
\hline Kinematic viscosity $\left(\mathrm{mm}^{2} / \mathrm{s}\right)$ & 76.491 & 61.949 & 39.282 & 26.167 & 18.329 & 13.474 & 10.325 & 7.961 \\
Dynamic viscosity $\left(\times 10^{-2} \mathrm{~Pa} \cdot \mathrm{s}\right)$ & 6.74 & 5.458 & 3.461 & 2.55 & 1.615 & 1.187 & 0.909 & 0.701 \\
\hline
\end{tabular}

$$
\begin{gathered}
\rho c_{p}\left(\frac{\partial T}{\partial t}+v_{r} \frac{\partial T}{\partial r}+\frac{v_{\theta}}{r} \frac{\partial T}{\partial \theta}+v_{z} \frac{\partial T}{\partial z}\right)= \\
k\left(\frac{\partial^{2} T}{\partial r^{2}}+\frac{1}{r} \frac{\partial T}{\partial r}+\frac{1}{r^{2}} \frac{\partial^{2} T}{\partial \theta^{2}}+\frac{\partial^{2} T}{\partial z^{2}}\right)+2 \mu\left[\left(\frac{\partial v_{r}}{\partial r}\right)^{2}+\right. \\
\left(\frac{1}{r} \frac{\partial v_{\theta}}{\partial \theta}+\frac{v_{r}}{r}\right)^{2}+\left(\frac{\partial v_{z}}{\partial z}\right)^{2}+\frac{1}{2}\left(\frac{\partial v_{\theta}}{\partial r}+\frac{1}{r} \frac{\partial v_{r}}{\partial \theta}-\frac{v_{\theta}}{r}\right)^{2}+ \\
\left.\frac{1}{2}\left(\frac{1}{r} \frac{\partial v_{z}}{\partial \theta}+\frac{\partial v_{\theta}}{\partial z}\right)^{2}+\frac{1}{2}\left(\frac{\partial v_{r}}{\partial z}+\frac{\partial v_{z}}{\partial r}\right)^{2}\right]
\end{gathered}
$$

where $T$ is the temperature, $c_{p}$ is the specific heat at constant pressure, $k$ is the thermal conductivity of the oil film [20].

\subsection{Viscosity-temperature relation}

For hydro-viscous drive, the most quantitatively significant dependence of any physical property is that of the viscosity with temperature. In this study, synthetical laboratory measurements and analysis were made on two kinds of lubricating oil (i. e. 8 \# hydrodynamic transmission oil and 32\# hydrodynamic transmission oil). The viscosity-temperature performance, along with experimental viscosities and the computed viscosities for the same oil were compared in the database. In addition, all measurements were conducted at the standard atmospheric environment. The experimental results showed that both the data from experimental kinematic viscosities and computed dynamic viscosities of $8 \#$ hydrodynamic transmission oil were larger than that of 32\# hydrodynamic transmission oil. Then $8 \#$ hydrodynamic transmission oil is more suitable for applications and popularize of HVD [21]. The fitting curve of viscosity versus temperature based on experimental viscosities as listed in Table 1 is shown in Fig. 2. The viscosity versus temperature equation is given by

$$
\eta=0.1472 e^{-0.0351 T}
$$

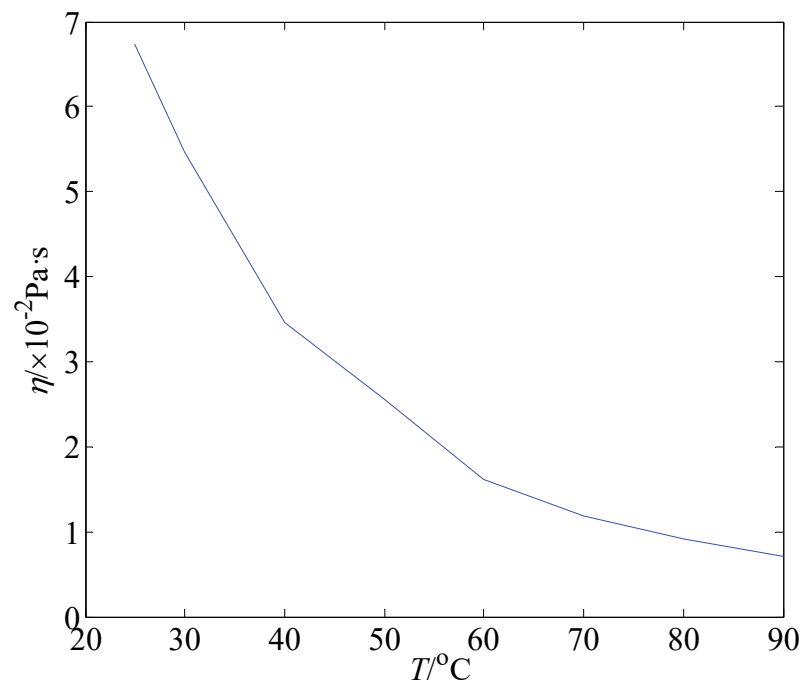

Fig. 2 Curve fit of viscosity-temperature relation

\subsection{Temperature distribution model}

In order to investigate the effect of temperature distribution and temperature difference from the friction pair on dynamic transmission, four forms of temperature distribution models (i. e. M1, M2, M3 and M4) are assumed to be put forward, as shown in Fig. 4. In Fig. 4(a), the highest temperature in M1 model are the same as that in M2 model, except for the different locations of two peaks along the radial direction. Figure 4(b) shows the two models of M3 and M4 along the radial direction. There exists some difference about peak value between two models. In order to better understand the effect of four models on dynamic transmission in HVD, concrete temperature models based on the parameters for simulation in Table 2 are adopted.

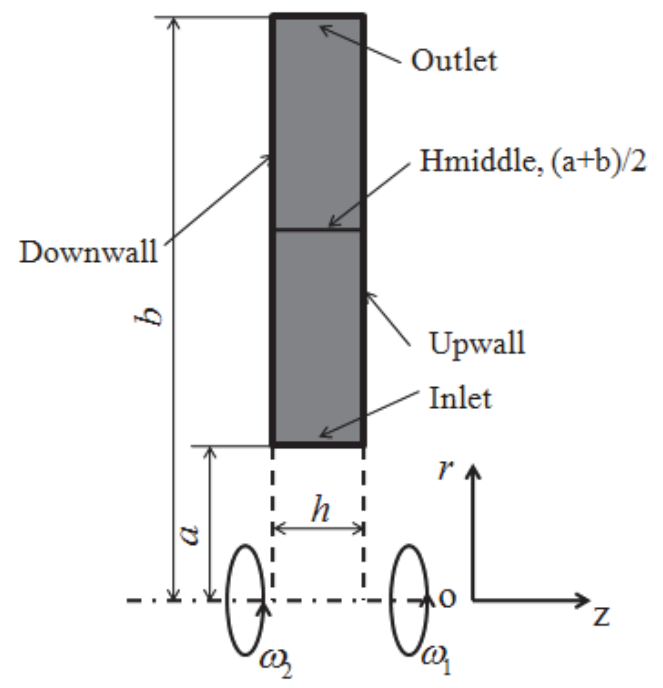

Fig. 3 Schematic of oil film between the disks of HVD

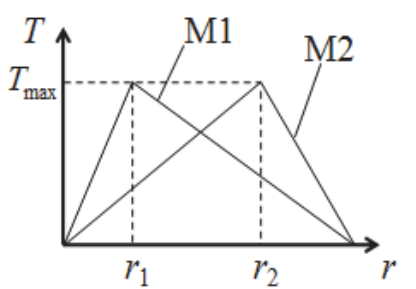

(a) M1-M2 model

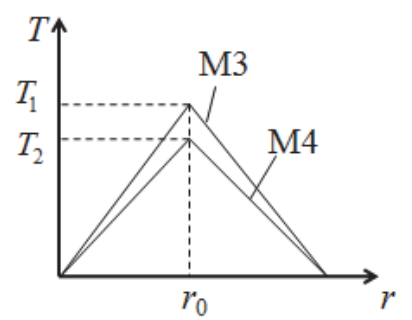

(b) M3-M4 model

Fig. 4 Four forms of temperature distribution models 
Table 2 Parameters for simulation

\begin{tabular}{lc}
\hline \multicolumn{1}{c}{ Parameters } & Value \\
\hline Inner radius $a /(\mathrm{m})$ & 0.09 \\
Outer radius $b /(\mathrm{m})$ & 0.125 \\
Oil film thickness $h /(\mathrm{mm})$ & 0.012 \\
Film density $\rho /\left(\mathrm{Kg} / \mathrm{m}^{3}\right)$ & 881.6 \\
Input speed $\omega_{1} /\left(\mathrm{r} \cdot \mathrm{min}^{-1}\right)$ & 1500 \\
Output speed $\omega_{2} /\left(\mathrm{r} \cdot \mathrm{min}^{-1}\right)$ & 105 \\
Inlet film flow rate $Q /\left(\mathrm{L} \cdot \mathrm{min}^{-1}\right)$ & 0.002 \\
Specific heat at constant $\mathrm{pressure} c_{p} /\left(\mathrm{J} / \mathrm{Kg} \cdot \mathrm{K}^{-1}\right)$ & 2000 \\
Thermal conductivity $\mathrm{k} /\left(\mathrm{W} / \mathrm{m} \cdot \mathrm{K}^{-1}\right)$ & 0.125 \\
\hline
\end{tabular}

$$
\begin{aligned}
& \mathrm{M1}:\left\{\begin{array}{l}
T=400 r+287,0.09 \leq r<0.1 \\
T=-160 r+343,0.1 \leq r \leq 0.125
\end{array}\right. \\
& \text { M2: }\left\{\begin{array}{l}
T=160 r+308.6,0.09 \leq r<0.115 \\
T=-400 r+373,0.115 \leq r \leq 0.125
\end{array}\right. \\
& \text { M3: }\left\{\begin{array}{l}
T=900 r+242,0.09 \leq r<0.11 \\
T=-1200 r+473,0.11 \leq r \leq 0.125
\end{array}\right. \\
& \text { M4: }\left\{\begin{array}{l}
T=600 r+269,0.09 \leq r<0.11 \\
T=-800 r+423,0.11 \leq r \leq 0.125
\end{array}\right.
\end{aligned}
$$

where both of the peak temperature from M1 model and M2 model are $327 \mathrm{~K}$ and two of them are located at $r_{1}=0.1 \mathrm{~m}$ and $r_{2}$ $=0.115 \mathrm{~m}$ respectively. Also, both of the peak temperature from M3 model and M4 model are located at $r_{0}=0.11 \mathrm{~m}$, in which the peak values in M3 model and M4 model are 335K and 341K respectively.

\section{Numerical simulation}

3.1 Geometrical model and mesh generation

In order to investigate the dynamic behavior of oil film between the disks in hydro-viscous drive, the effect of groove on the flow field is neglected during the soft-start. A single pair of friction and separator disks is considered to reduce the run time of the model. And two-dimensional simulation model is taken into account due to the axisymmetric structure of the disks. As shown in Fig. 3, the gray area indicates the fluid computational domain. The two identical annular discs with inner radius $a$ and outer radius $b$ are separated by a distance $h$ along the direction of the rotation axis $z$. The left disk and the right disk rotate with angular velocity $\omega_{2}$ and $\omega_{1}$, respectively. Due to the non-slip condition at the surface of the disks, the rotation of the disks sets the fluid in the gap in motion. For this reason, the wall surface of the driving disk is named Upwall with the same speed as the oil film, as well as the Downwall for the driven disk. Inlet and Outlet in the figure correspond to the boundaries of the inlet and outlet for the oil film, respectively. Hmiddle, $r=(a+b) / 2$, represents the intermediate layer of the oil film along the radial direction.

The energy equation is coupled with momentum equation each other due to variable viscosity with temperature. As a result, momentum and energy equations could be solved analytically, even though they have been simplified. Consequently, the flow is solved numerically by using computational fluid dynamics (CFD) code FLUENT based on finite volume method (FVM) in this case. The physical model was built in CFD pre-processing code GAMBIT. Because of the comparative advantage, the mesh generation method about structured quadrilateral grid was used to mesh the model. Furthermore, since the gap between the two disks is very small compared to their radii, there are great gradients of shear stress and velocity along $z$-direction due to large velocity difference between the disks [18]. To reduce the grid size and improve computational efficiency, the influence of mesh number on the simulation accuracy is analyzed. In the present paper, the simulation test that depends on the variation of viscous torque is conducted to deal with numerical errors and loss of computational efficiency. Based on the relationship between viscosity and temperature, the grid density is not adjusted until viscous torque acting on the driven disk remains nearly unchanged. The simulation results were found that the effect of the number of grids on viscous torque could be neglected, as presented in Table 3. As a result, a high-resolution grid employing 466700 cells was used as the reference model.

\subsection{Boundary conditions and solution procedure}

Fluid is introduced through a central conduit and directly flows from the inner to the outer radii of the annular disks. As shown in Fig. 3, the boundary marked with "inlet" is specified as velocity inlet boundary condition. The boundary marked with "outlet" is specified as pressure outlet boundary condition, and the pressure value is assigned to be zero. The two boundaries marked with "Upwall" and "Downwall" are specified as temperature boundary conditions from four forms of temperature distribution models.

The solver and parameters related to numerical simulation as listed in Table 2 were specified in FLUENT. An axisymmetric swirl calculation model was chosen in FLUENT. Pressurevelocity coupling was resolved by means of SIMPLEC algorithm. The second order upwind was used to discretize the momentum and energy equations. Since the viscosity of the oil film is greatly influenced by the temperature, an UDF routine about the fitting function is constructed and compiled to involve iterative computations [22]. The procedure of numerical simulation is as follows: firstly, the pressure and temperature distribution in the radial direction are simulated numerically. And then, heat transfer equation and viscosity-temperature equation are added to the governing equations. The pressure and temperature distributions are numerically simulated respectively. Lastly, Newton power-law equation is used as fluid constitutive equation, and the pressure and temperature distributions are computed.

\section{Results and discussion}

4.1 Temperature distribution

The results of the numerical solution about temperature

Table 3 Grid independence verification

\begin{tabular}{ccc}
\hline No & Grid number & Viscous torque $(\mathrm{N} \cdot \mathrm{m})$ \\
\hline 1 & 466600 & 75.656 \\
2 & 466650 & 75.658 \\
3 & 466700 & 75.660 \\
4 & 490035 & 75.662 \\
5 & 513370 & 75.664 \\
\hline
\end{tabular}




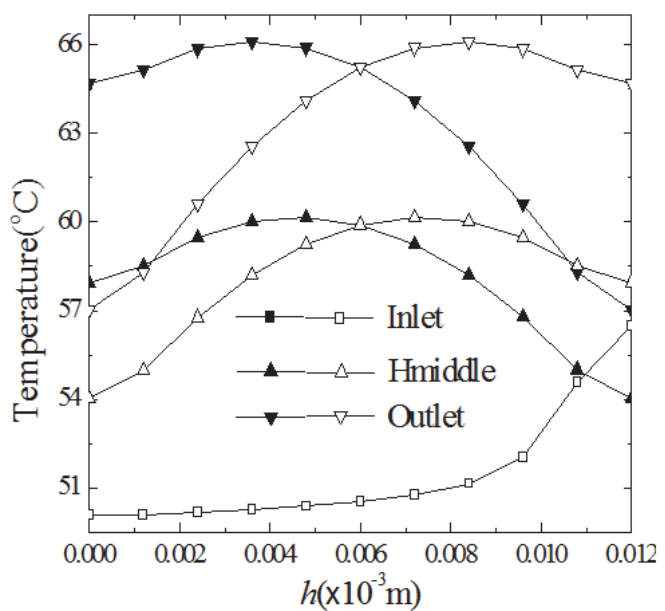

(a) Non-isothermal (M1-M2 model)

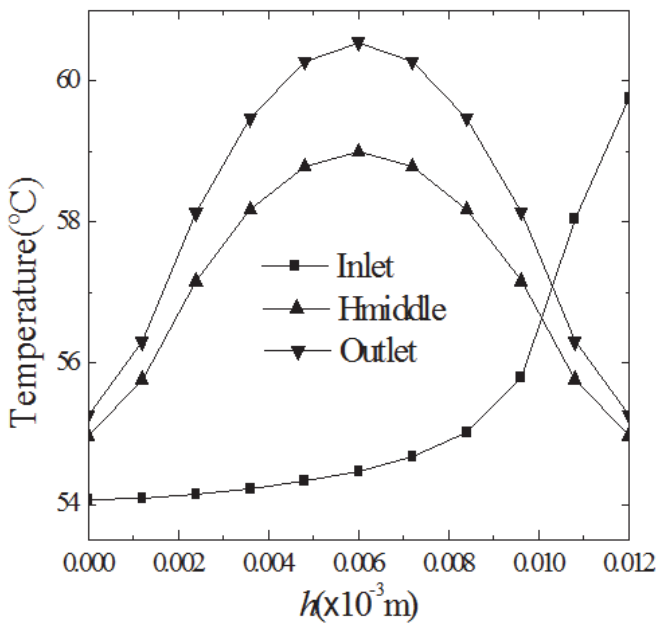

(b) Isothermal

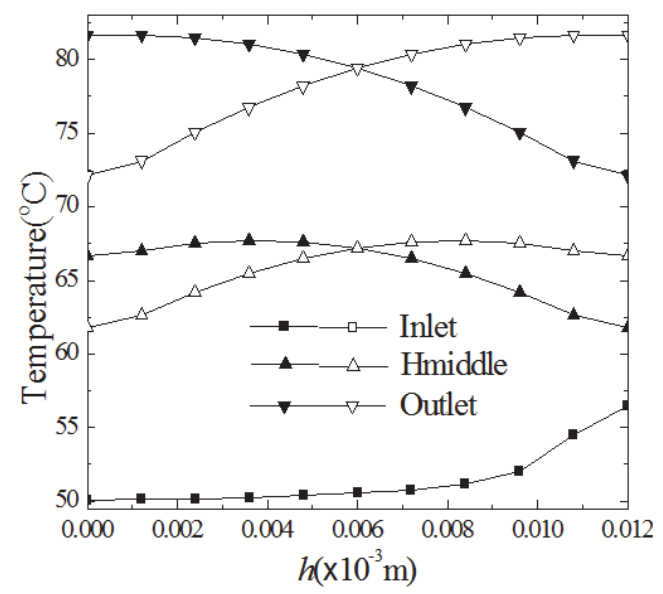

(c) Non-isothermal (M3-M4 model)

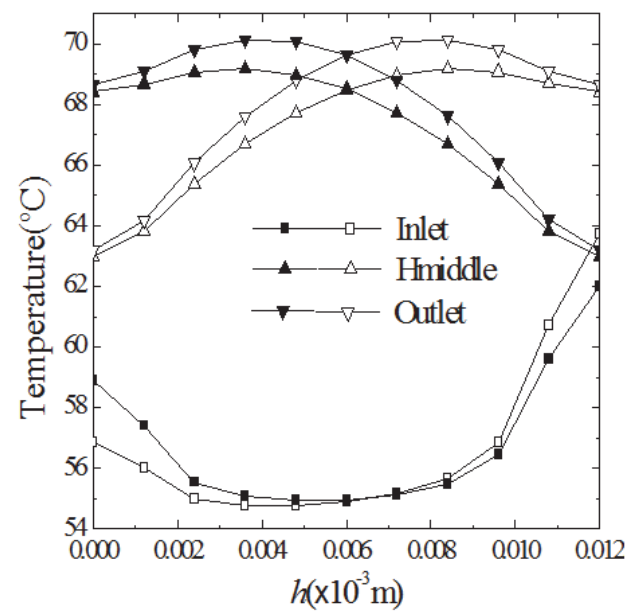

(d) Isothermal

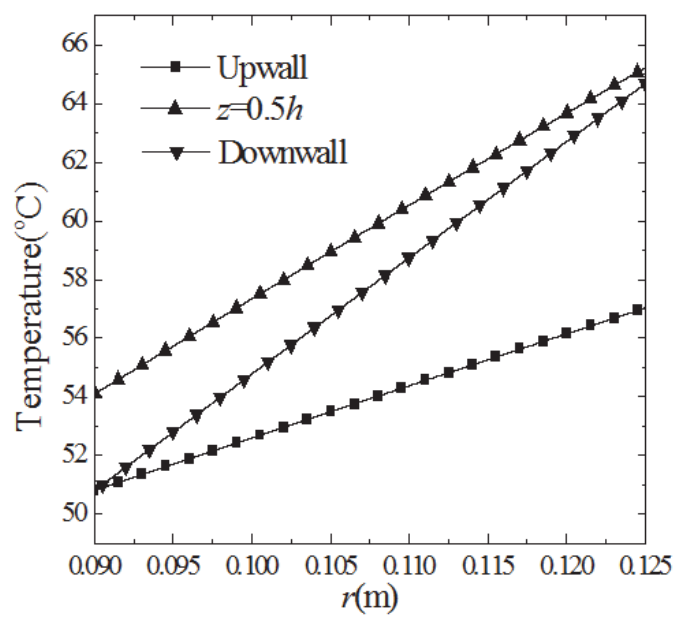

(e) M1downM2up model

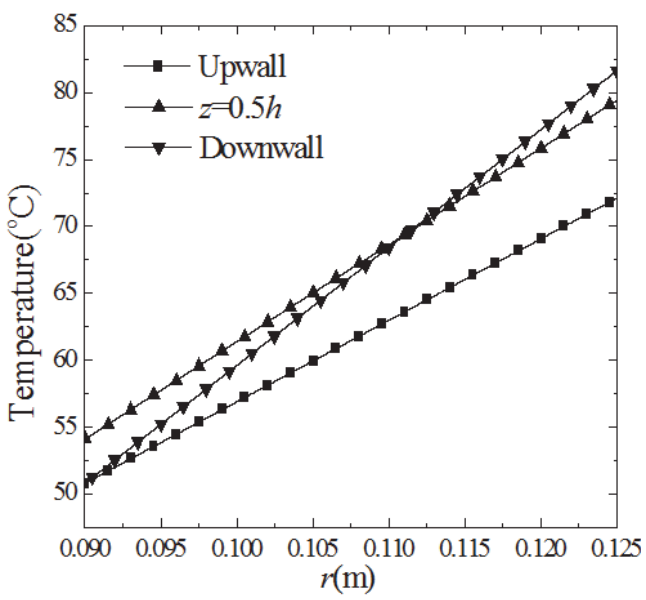

(f) M3downM4up model

Fig. 5 Temperature distribution

distribution of the oil film along $r$-direction are shown in Fig. 5 . Figure 5(a) and (b) compare temperature profiles under two kinds of boundary conditions, i. e. non-isothermal interfaces for M1-M2 model and isothermal interfaces for $T=327 \mathrm{~K}$. Also, Fig. 5(c) and (d) show a comparison between non-isothermal interfaces for M3-M4 model and isothermal interfaces for $T=$ $335 \mathrm{~K}$ and $T=341 \mathrm{~K}$. As shown in Fig. $5(\mathrm{a})$ and (c), the solid lines denote temperature profiles when the wall boundaries are set as
M1downM2up model and M3downM4up model respectively. Similarly, the hollow lines denote the results based on M1upM2down model and M3upM4down model respectively. Figure 5(e) and (f) show temperature distribution along $r$-direction based on M1downM2up model and M3downM4up model respectively. It should be noted that M1downM2up, M1upM2down, M3downM4up and M3upM4down mean that the moving Downwall or the moving Upwall is set as M1 model, 
M2 model, M3 model and M4 model, respectively.

When the two moving walls are set as the same peak temperature located at different radius, it can be found that temperature profiles at the outlet can be depicted as asymmetric parabolic curves, which is different from the ideal parabolic curves with isothermal interfaces. Furthermore, take the operating condition of M1downM2up as an example, as peak temperature appears at the inner radius of the Downwall and the outer radius of the Upwall, the highest temperature at the outlet is on the verge of the driven disk. This is because the oil film near the outer radius of the driving disk has smaller viscosity and larger radial velocity than that near the outer radius of the driven disk. Therefore, temperature rise near the wall of the driving disk is smaller than that of the driven disk.

On the other hand, if the operating condition of M3downM4up is applied, the two moving walls are set as different peak temperature located at the same radius. Average temperature near the wall of the driven disk is larger than that of the driving disk. However, compared with the heat transfer effect between fluid and wall, the cooling effect of mass flow caused by larger radial velocity is deemed small. Thus, the highest temperature at the outlet is prone to the driven disk.

In addition, when the wall boundaries are set as the same temperature along the radial direction, it is found that the highest temperature occurs at $z=0.5 h$, as shown in Fig. 5(b). Moreover, as shown in Fig. 5(d), temperature difference between the two wall boundaries also leads to asymmetrical temperature distribution. However, there is obvious temperature difference about peak value in spite of the same operating conditions. This

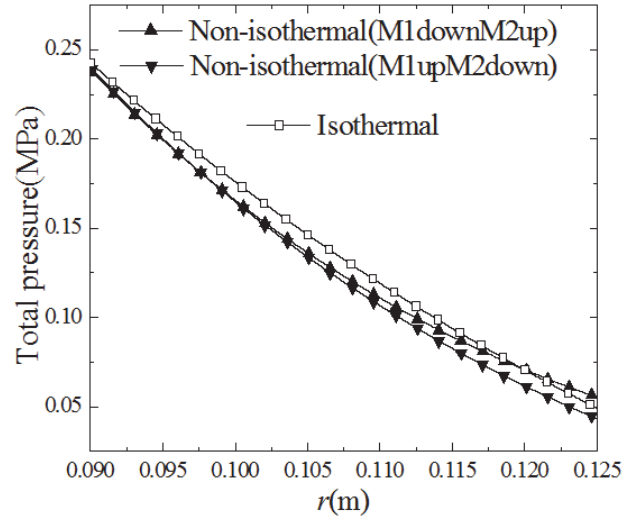

(a) Comparison of non-isothermal (M1-M2 model) and isothermal

is due to the fact that frictional heat generated by the shear of oil film increases the temperature gradient both along $z$-direction and along $r$-direction. Also, non-isothermal temperature distribution of wall boundaries of the oil film is responsible for the development of a typically non-uniform temperature field.

\subsection{Pressure distribution}

To investigate the effect of four boundary conditions on the film pressure, radial film layer at $z=0.5 h$ is applied as the analysis of the object. As shown in Fig. 6, the pressure distribution along $r$-direction between M1-M2 model and M3-M4 model is compared. It is obvious that the pressure profiles in the boundary conditions of M1downM2up and M1upM2down show a similar agreement as compared to those of M3downM4up and M3upM4down. For M1-M2 model, film pressure at the outlet based on the boundary condition of M1downM2up is larger than that of M1upM2down. This in fact reflects the discrepancy about film viscosity between two boundary conditions. When the temperature of wall boundaries does not change with radius, the impact on pressure is less pronounced when compare to the case of non-isothermal boundary conditions.

\subsection{Velocity distribution}

Figures 7 and 8 present radial velocity profiles and tangential velocity profiles at $z=0.5 \mathrm{~h}$ of the oil film under both the effect of non-isothermal interfaces and isothermal interfaces. In Fig. 7, it can be found that radial velocity decreases gradually as radius increases, which have the same changed trend as pressure

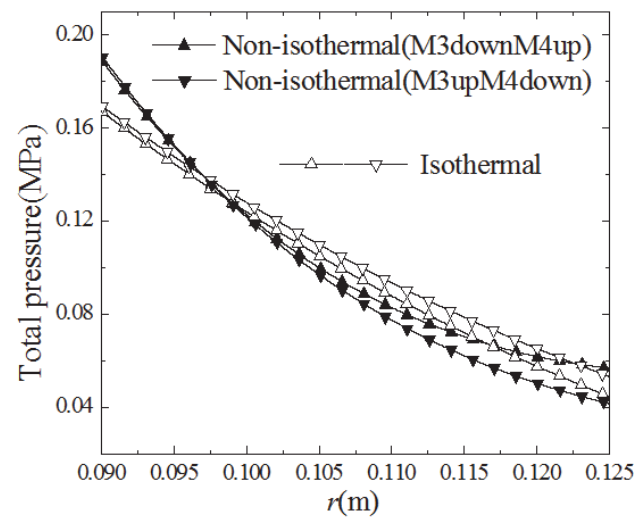

(b) Comparison of non-isothermal (M3-M4 model) and isothermal Fig. 6 Total pressure along $r$-direction $(z=0.5 h)$

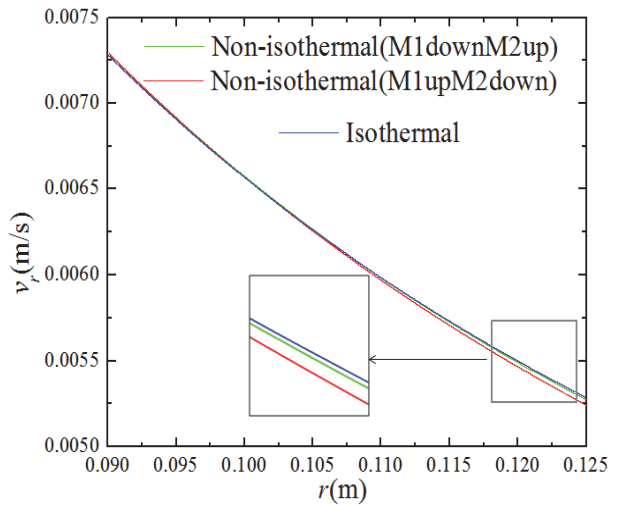

(a) Comparison of non-isothermal (M1-M2 model) and isothermal

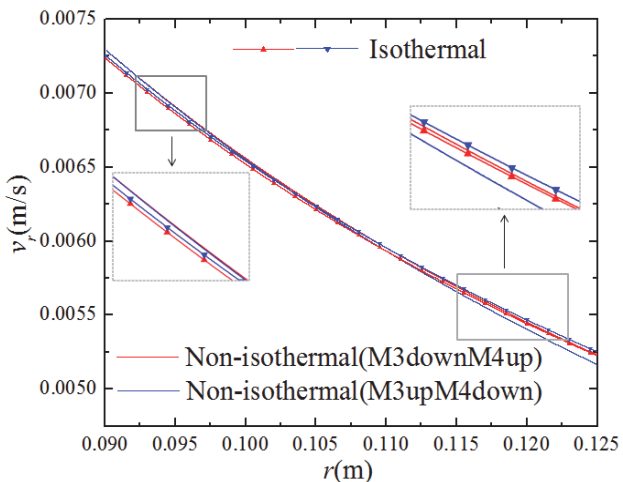

(b) Comparison of non-isothermal (M3-M4 model) and isothermal

Fig. 7 Radial velocity along $r$-direction $(z=0.5 h)$ 


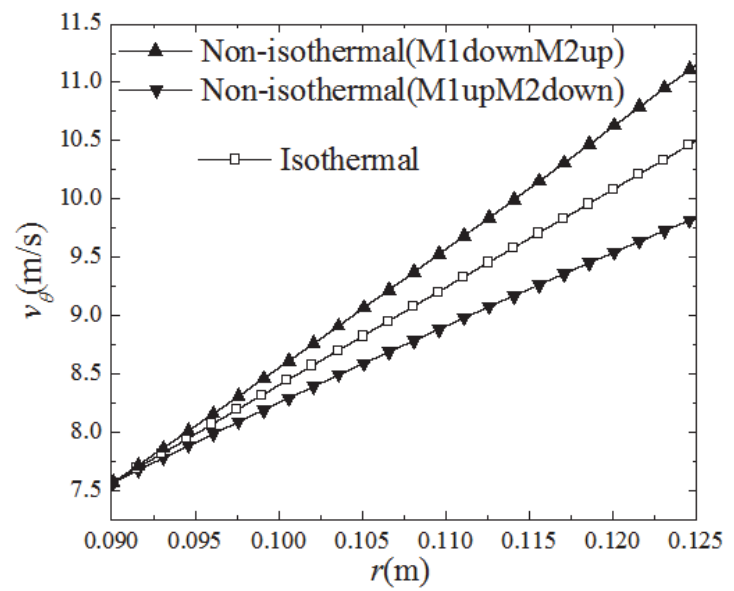

(a) Comparison of non-isothermal (M1-M2 model) and isothermal

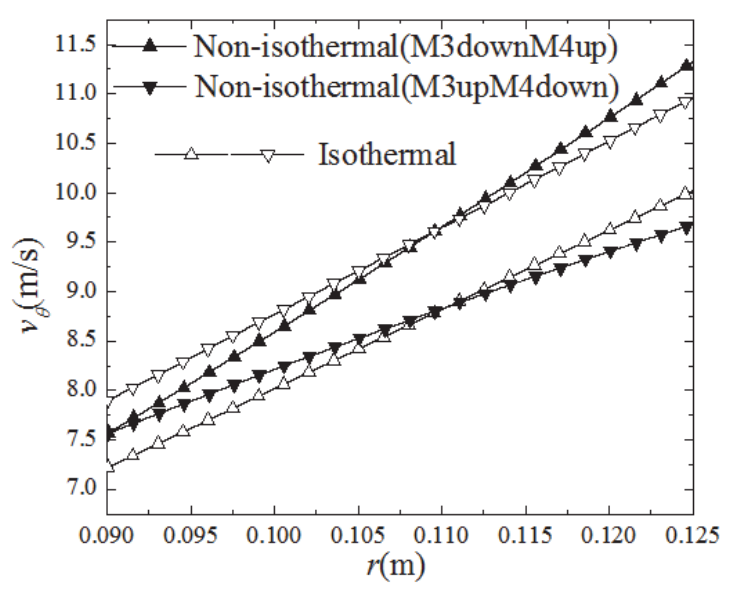

(b) Comparison of non-isothermal (M3-M4 model) and isothermal Fig. 8 Tangential velocity along $r$-direction $(z=0.5 h)$

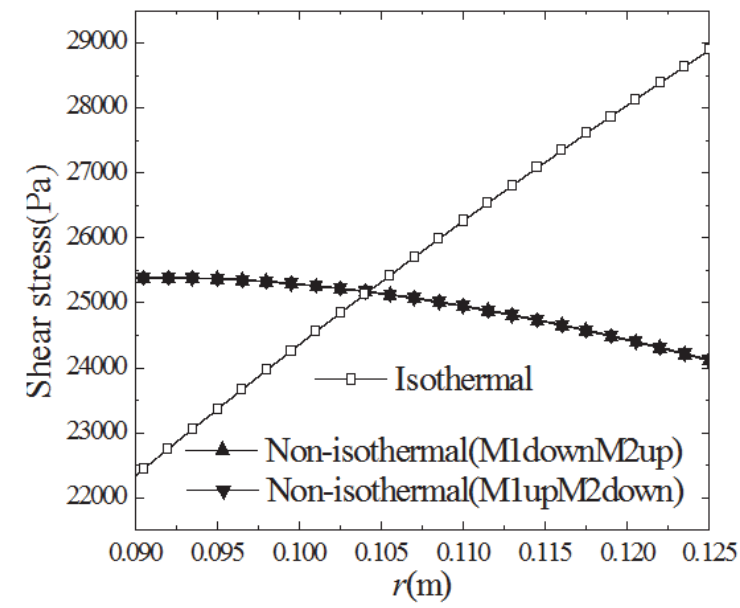

(a) Comparison of non-isothermal (M1-M2 model) and isothermal

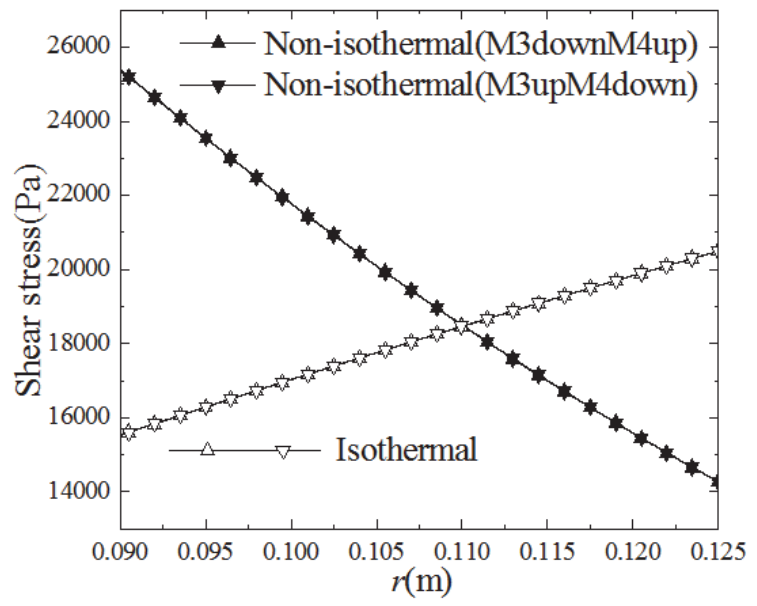

(b) Comparison of non-isothermal (M3-M4 model) and isothermal the driven disk

Table 4 Viscous torque/(N·m)

\begin{tabular}{ccccc}
\hline & M1downM2up & M1upM2down & M3downM4up & M3upM4down \\
\hline Viscous torque & 63.758 & 63.800 & 48.382 & 48.376 \\
\hline
\end{tabular}

profiles. With four kinds of non-isothermal boundary conditions, the increase of tangential velocity appears some differentiation along $r$-direction, which results from the changes of viscosity. It seems that temperature distribution about the interfaces has no effect on radial velocity except the outlet. The study also includes finding almost the same distribution pattern in which non-isothermal interfaces has limited effect on tangential velocity, as shown in Fig. 8. It is obvious that as radius increases, the effects of non-isothermal interfaces begin to come out. Oil viscosity changes as a result of changes in oil temperature, so the tangential velocity profiles show large difference as compared to the radial velocity profiles. In conclusion, under the described boundary conditions, the simulation results from M1-M2 model to M3-M4 model show a large difference with the pressure profiles, so additional considerations need to be made to reduce turbulence at the outlet.

\subsection{Shear stress and viscous torque distribution}

Figure 9 shows the effect of shear stress on the driven disk under different thermal boundary conditions. For
M1downM2up boundary condition or M1upM2down boundary condition, there is a curved decreasing tendency about the shear stress along the radial direction. Also, notice that shear stress decreases with the increase of the radius based on the M3-M4 model. The numerical solution is different from the results obtained by J. H. Huang et al. [10]. It is clear that while the isothermal boundary conditions are adopted, as the radius increases, the oil viscosity change slightly and tangential velocity increases gradually, which leads to the increase of shear stress. However, due to the non-isothermal boundary conditions, the shear stress profiles show the opposite trend caused by the substantial variation about the oil viscosity. On the other hand, as shown in Fig. 9(a), shear stress decreases at a slower pace at the inlet and then drops sharply. This means that the shear stress depends strongly on the distribution of peak temperature. Furthermore, the viscous torque values obtained show good agreement with the shear stress profiles, as shown in Table 4. The minor difference in numerical calculation can be neglected if there is no urgent need for high precision hydroviscous drive. 


\section{Experimental verification}

Figure 10 shows an experimental apparatus which was set up to allow measurements of the dynamic characteristics between non-isothermal disks. One plate was rotated with a variable speed motor and the other was fixed in place and instrumented with a torque-speed sensor and magnetic powder brake to measure any torque resulting from the fluid coupling between the gap. Hydraulic oil was pumped from a sump into the gap between the disks through a passage on the axis of the rotating disk's shaft. A syringe pump was used to carefully meter the oil flow and the inlet oil pressure was monitored with an analog pressure gauge. Thermocouples were used to monitor oil temperature as it enters the disks and when it is ejected from the outer radius. Also, a heater was used to warm the oil if desired.

In the present study, the surface temperature of the driving disk is set as constant due to the limitation of measurement conditions. Furthermore, integral electric heating disk and joint electric heating disk are used as the driven disks to test the effects of non-isothermal interfaces. In the experiments, three sampling points $(r=0.1 \mathrm{~m}, r=0.11 \mathrm{~m}$ and $r=0.12 \mathrm{~m})$ in the flow are distributed along the $r$-direction. Temperature and pressure are measured with platinum resistance temperature sensor Pt100/A and dynamic pressure sensor respectively. Then overall temperature rise in the integral electric heating disk is precisely controlled by temperature control system. The temperature of the integral electric heating disk is kept at $325 \mathrm{~K}, 327 \mathrm{~K}$ and $330 \mathrm{~K}$ respectively while it is applied to the non-isothermal interfaces. Then it is undoubtedly that temperature difference between non-isothermal disks lead to the increase of oil temperature and the decrease of oil pressure adjacent to the driven disk. In addition, stepped temperature rise is available in the joint electric heating disk on condition that all the joints are insulated.

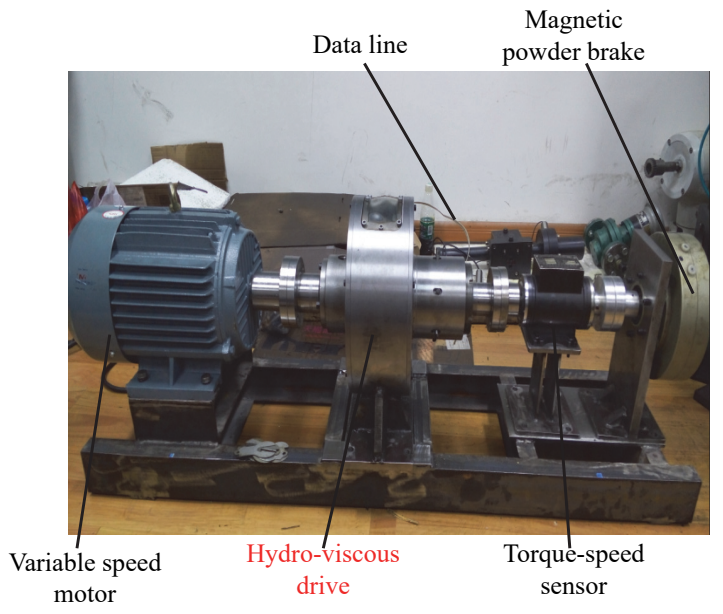

Fig. 10 Experimental setup of hydro-viscous drive system

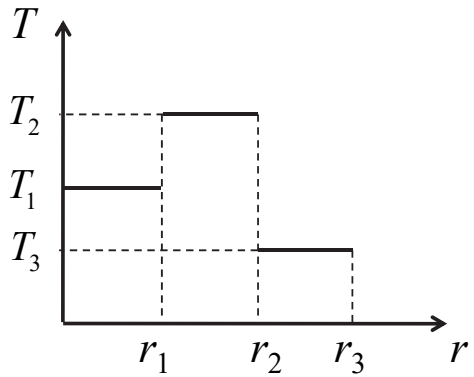

Fig. 11 Temperature setting of driven disk
Figure 11 shows the temperature setting in the $r$-direction about the driven disk. For the measured curves from experimental apparatus in Fig. 12, the boundary with lower temperature at the outer radius results in the increase of oil temperature and the decrease of oil pressure. It can be seen that the experimental results agree well with the simulation results. Finally, for the torque profiles in Table 5, the experimental results are mainly affected by the temperature difference at the outer radius.

\section{Conclusions}

The flow field between non-isothermal interface in hydroviscous is investigated for the proper simulation modeling of the dynamic behavior of the oil film. Based on the computation fluid dynamics code FLUENT, an improved CFD modeling procedure is developed with consideration of four forms of

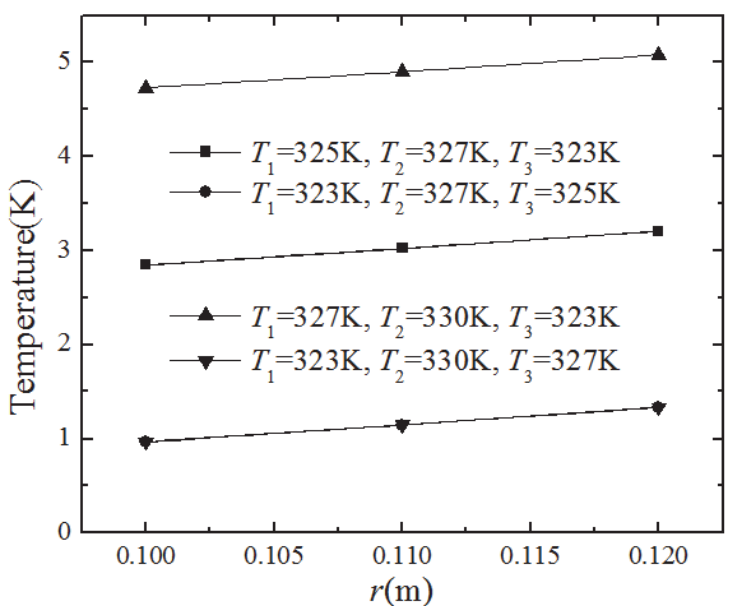

(a) Temperature

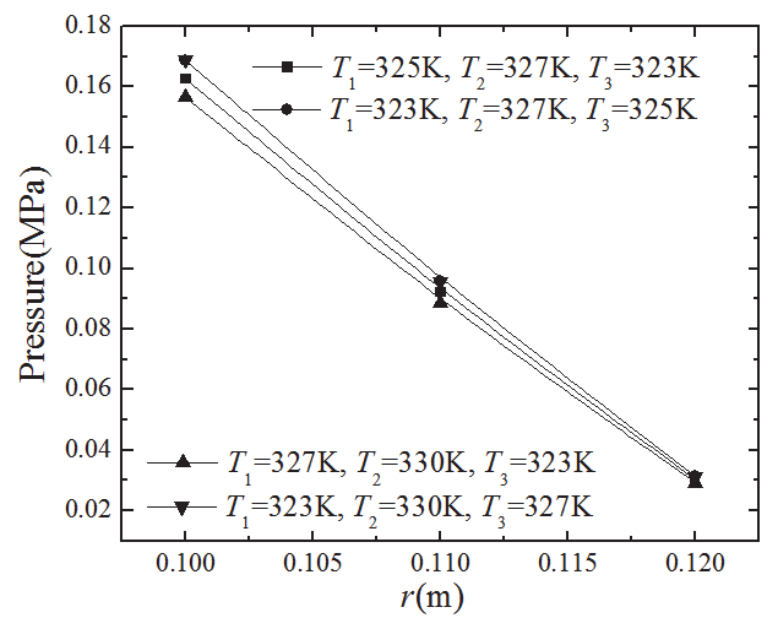

(b) Pressure

Fig. 12 Measured curves from experimental apparatus

Table 5 Measured torque/(N.m)

\begin{tabular}{cc}
\hline Temperature distribution of the driven disk & Value \\
\hline$T_{1}=325 \mathrm{~K}, T_{2}=327 \mathrm{~K}, T_{3}=323 \mathrm{~K}$ & 73.278 \\
$T_{1}=323 \mathrm{~K}, T_{2}=327 \mathrm{~K}, T_{3}=325 \mathrm{~K}$ & 75.665 \\
$T_{1}=327 \mathrm{~K}, T_{2}=330 \mathrm{~K}, T_{3}=323 \mathrm{~K}$ & 70.871 \\
$T_{1}=323 \mathrm{~K}, T_{2}=330 \mathrm{~K}, T_{3}=327 \mathrm{~K}$ & 75.715 \\
\hline
\end{tabular}


temperature distribution models. The following conclusions are achieved:

(1) Compared with isothermal interface from hydro-viscous drive, non-isothermal interface results in temperature distribution profiles that diverge from the ideal parabolic curve. Similarly, both of the discrepancies about film pressure and radial velocity at the outlet are vulnerable to temperature variation derived from a set of frictional pair, which reflects actual working conditions especially when each set of frictional pair suffers from different cooling effects.

(2) Due to the effect of non-isothermal interface, tangential velocity profiles diverge from inlet to outlet. Shear stress profiles show the opposite trend caused by the substantial variation about the oil viscosity. Viscous torque depends strongly on the distribution of peak temperature. It can be inferred that the more the number of friction pairs, the greater the error of transmission torque.

\section{Acknowledgments}

This work is supported by grants from Scientific Research Foundation of Advanced Professional of Yancheng Institute of Technology (XJ201701) , Project funded by China Postdoctoral Science Foundation (NO. 2018M632199) and the National Natural Science Foundation of China (No. 51675234).

\section{Nomenclature}

$a, b=$ inner, outer radius of disk, respectively

$\omega_{1}, \omega_{2}=$ angular velocity of driving and driven plate, respectively

$h=$ average film thickness

$v_{\theta}, v_{r}=$ tangential velocity and radial velocity, respectively

$Q=$ inlet film flow rate

$\eta=$ dynamic viscosity of lubricating oil

$\rho=$ oil density

$\tau_{z \theta}=$ shear stress acting on the driven disk

$T_{h}=$ viscous torque acting on the driven disk

$T=$ oil temperature

$c_{p}=$ specific heat at constant pressure

$k=$ thermal conductivity of the oil film

$p=$ oil pressure

\section{References}

[1] Xie, F. W. and Hou, Y. F., "Oil Film Hydrodynamic Load Capacity of Hydro-Viscous Drive with Variable Viscosity," Industrial Lubrication and Tribology, 63, 3, 2011, 210-215.

[2] Cui, J. Z., Wang, C. T., Xie, F. W., Xuan, R. and Shen, G., “Numerical Investigation on Transient Thermal Behavior of Multidisk Friction Pairs in Hydro-Viscous Drive," Applied Thermal Engineering, 67, 1-2, 2014, 409-422.

[3] Yevtushenko, A. A. and Grzes, P., “3D FE Model of Frictional Heating and Wear with a Mutual Influence of the Sliding Velocity and Temperature in a Disc Brake," International Communications in Heat and Mass Transfer, 62, 2015, 37-44.

[4] Liu, J., Wang, Y. S., Ke, L. L. and Yang, J., “Thermo-Elastic Dynamic Instability of an Elastic Half-Plane Sliding against a Coated HalfPlane," International Journal of Mechanical Sciences, 117, 2016, 275285.
[5] Davis, C. L., Sadeghi, F. and Krousgrill, C. M., “A Simplified Approach to Modeling Thermal Effects in Wet Clutch Engagement: Analytical and Experimental Comparison," ASME J. Tribol., 122, 1, 2000, 110-118.

[6] Berger, E. J., Sadeghi, F. and Krousgrill, C. M., "Analytical and Numerical Modeling of Engagement of Rough, Permeable, Grooved Wet Clutches," ASME J. Tribol., 119, 1, 1997, 143-148.

[7] Yuan, Y. Q., Attibele, P. and Dong, Y., "CFD Simulation of the Flows Within Disengaged Wet Clutches of an Automatic Transmission," SAE Technical Paper, 2003, 2003-01-0320.

[8] Aphale, C. R., Cho, J. Y., Schultz, W. W., Ceccio, S. L., Yoshioka, T. and Hiraki, H., "Modeling and Parametric Study of Torque in Open Clutch Plates," ASME J. Tribol., 128, 2, 2006, 422-430.

[9] Yuan, Y. Q., Liu, E. A., Hill, J. and Zou, Q., “An Improved Hydrodynamic Model for Open Wet Transmission Clutches," ASME J. Fluids Eng., 129, 3, 2007, 333-337.

[10] Huang, J. H., Qiu, M. X., Liao, L. L. and Fu, L. J., “Numerical Simulation of Flow Field between Frictional Pairs in Hydroviscous Drive Surface," Chin. J. Mech. Eng., 21, 3, 2008, 72-75.

[11] Huang, J. H., Wei, J. H. and Qiu, M. X., “Laminar Flow in the Gap between Two Rotating Parallel Frictional Plates in Hydro-Viscous Drive," Chin. J. Mech. Eng., 25, 1, 2012, 144-152.

[12] Xie, F. W., Hou, Y. F. and Yang, P., “Drive Characteristics of Viscous Oil Film Considering Temperature Effect," ASME J. Fluids Eng., 133, 4, 2011, 044502.

[13] Manshoor, B., Jaat, M., Izzuddin, Z. and Amir, K., “CFD Analysis of Thin Film Lubricated Journal Bearing," Procedia Eng., 68, 2013, 5662.

[14] Lin, Q. Y., Wei, Z., Wang, N. and Chen, W., "Analysis on the Lubrication Performances of Journal Bearing System Using Computational Fluid Dynamics and Fluid-Structure Interaction Considering Thermal Influence and Cavitation," Tribology International, 64, 2013, 8-15.

[15] Li, M., Khonsari, M. M., McCarthy, D. M. C. and Lundin, J., "Parametric Analysis for a Paper-Based Wet Clutch with Groove Consideration," Tribology International, 80, 2014, 222-233.

[16] Xiong, C. B., Ma, B., Li, H. Y., Zhang, F. L. and Wang, Y. S., “Modeling and Analysis of Transient Temperature Field in Finite Thickness Plate under Symmetrically Located Moving Heat Sources," Advances in Mech. Eng., 7, 11, 2015, 1-11.

[17] Zhigang, Z., Xiaohui, S. and Dong, G., "Dynamic Temperature Rise Mechanism and Some Controlling Factors of Wet Clutch Engagement," Mathematical Problems in Engineering, 2016, 6530213.

[18] Cho, J., Katopodes, N., Kapas, N. and Fujiii, Y., "CFD Modeling of Squeeze Film Flow in Wet Clutch," SAE Technical Paper, 2011, 201101-1236.

[19] Noh, D., Kim, S., Kim, Y. and Jang, J., "Lubricant Flow Analysis for Effective Lubrication of Tractor Forward/Reverse Clutch," Heliyon, 3, 4, 2017, e00295.

[20] Yang, L., Ma, B., Ahmadian, M., Li, H. and Vick, B., “Pressure Distribution of a Multidisc Clutch Suffering Frictionally Induced Thermal Load," Tribology Transactions, 59, 6, 2016, 983-992.

[21] Cui, J. Z., Xie, F. W., Wang, C. T., Zhang, X. J. and Xuan, R., “Dynamic Transmission Characteristics during Soft-Start of Hydro-Viscous Drive Considering Fluid-Inertia Item," Tribology Online, 10, 1, 2015, 35-47.

[22] Müller, M., Völpel, A. and Ostermeyer, G. -P., “On the Influence of Fluid Dynamics and Elastic Deformations on Pressure Buildup in Partially Filled Gaps," Tribology International, 105, 2017, 345-359. 